\title{
Bicaval orthotopic heart transplantation in a patient with persistent left superior vena cava
}

\author{
Diogo Luiz de Magalhães Ferraz, MD, Felipe Ribeiro Walter, MD, and \\ Cristiano Berardo Carneiro da Cunha, MD, MSc, Recife, Brazil
}

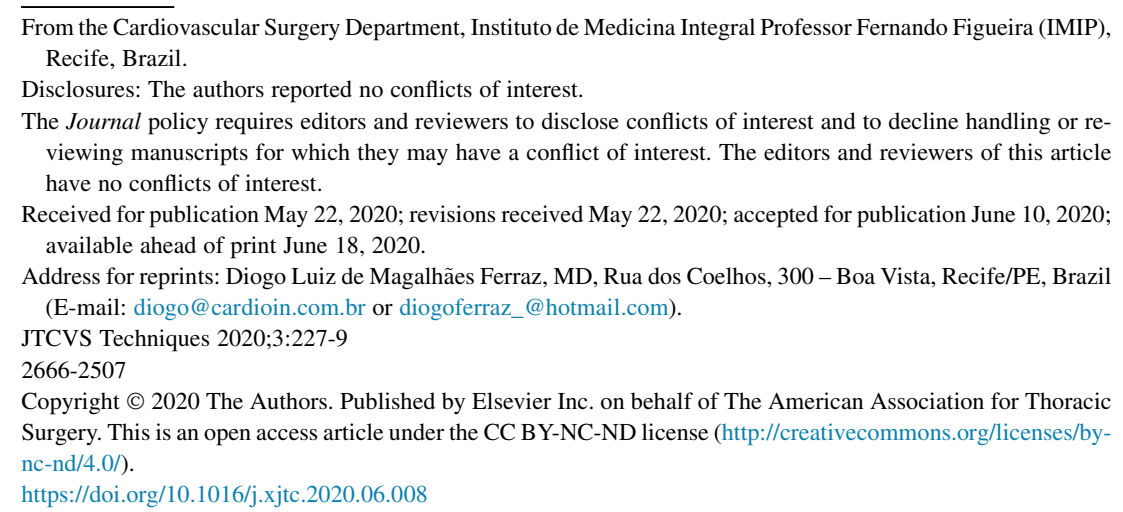

Video clip is available online.

The known incidence of persistent left superior vena cava (PLSVC) is $0.3 \%$ to $0.5 \%$. In patients with other congenital heart abnormalities, this rate increases to approximately $12 \%$. PLSVC may occur with many anatomical variations, the majority of cases are asymptomatic, and it is usually diagnosed during surgery. ${ }^{1,2}$

Written informed consent was obtained from the patient, and the study was approved by the institutional review board on May 13, 2020 (registration number [CAAE]: 31367720.5.0000.5201). A 21-year-old woman with idiopathic dilated cardiomyopathy and significant pulmonary arterial hypertension received a heart transplant in our department. An echocardiogram and chest computed tomography scan showed the PLSVC draining into the coronary sinus and absence of the right superior vena cava (Figure 1, $A$ and $B$ ).

Orthotopic heart transplantation was performed using the bicaval technique, by median sternotomy, cardiopulmonary bypass (CPB), using arterial cannulation in the ascending aorta with a 21-Fr cannula and inferior vena cava with a 30Fr cannula. Venous drainage from the upper body was performed with a 24-Fr cannula in the innominate vein (Video 1).

Cardiectomy was performed by transection of the pulmonary artery and ascending aorta. The right atrium was opened, preserving the PLSVC and the coronary sinus (Figure 2). The

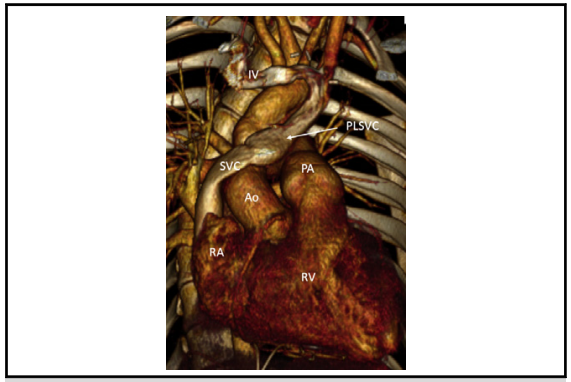

Persistent left superior vena cava anastomosed on superior vena cava of donor heart.

\section{CENTRAL MESSAGE \\ It was possible to perform a surgical technique for a bicaval orthotopic heart transplant without the use of prosthetic materials in a patient with persistent left superior vena cava.}

See Commentaries on pages 230 and 232.

left atrium was opened, and the incision was extended toward the mitral valve annulus, maintaining the pulmonary veins. At this point, the native heart was removed.

Because the PLSVC was diagnosed before the surgery, an extended length of superior vena cava (SVC) was removed, the azygos vein was ligated, and the innominate vein was harvested (Figure 2).

Heart implantation occurred in the usual sequence: (1) Left atrium. (2) The donor SVC was directly anastomosed in the PLSVC, in an anterior position to the great vessels. Part of the right atrium and the recipient's coronary sinus were used as a conduit for anastomosis (Figure 1,C). (3) Inferior vena cava. (4) Pulmonary artery. (5) Aorta. All the anastomoses were made with simple continuous sutures.

Due to high pulmonary vascular resistance, inhaled nitric oxide was used throughout the procedure and the patient required inotropic support with dobutamine, epinephrine, and milrinone at the separation from CPB. The CPB time was 150 minutes, and the crossclamp time was 190 minutes.

The immediate postoperative period was uneventful. The patient received standard immunosuppression. The patient 

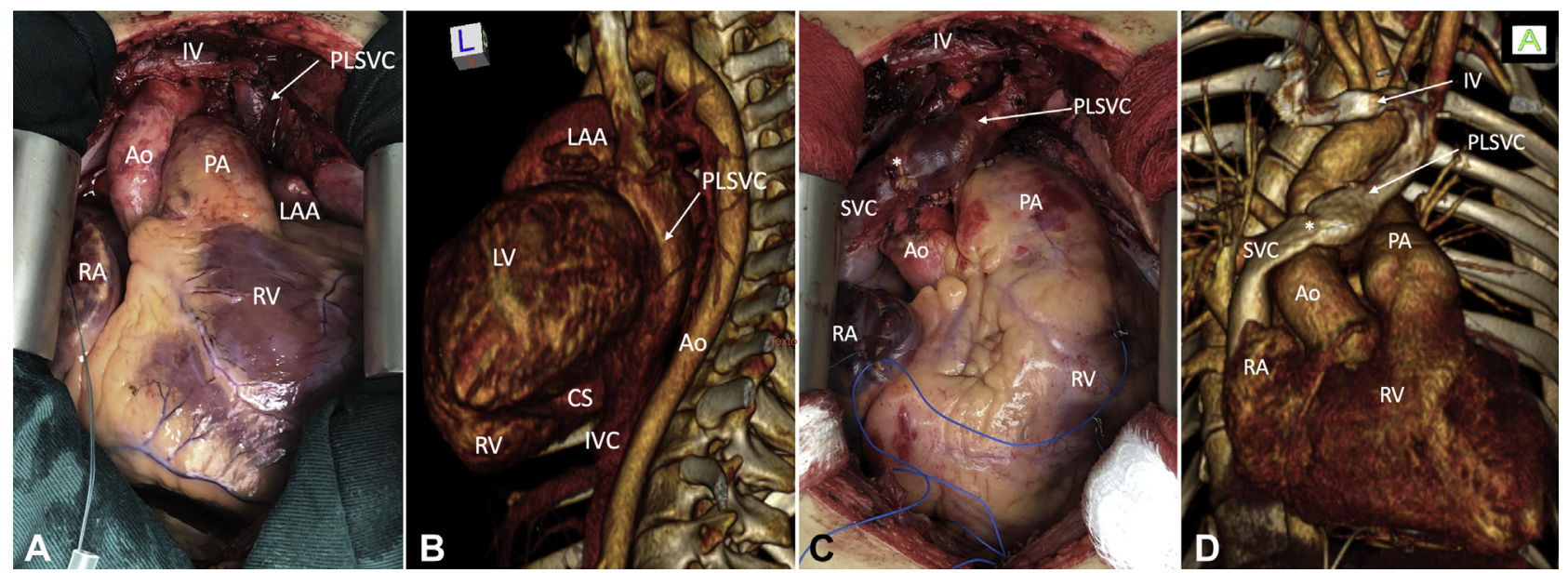

FIGURE 1. Heart transplantation with persistent left vena cava using the bicaval technique. Intraoperative photograph and CT 3D reconstruction. A, Native heart before explantation. B, The inferolateral view of PLSVC on CT. C, Surgical aspect after cardiac implantation. D, CT on the 30th postoperative day shows normal venous drainage through the PLSVC to the SVC. Asterisk: suture line. IV, Innominate vein; PLSVC, persistent left superior vena cava; Ao, aorta; $P A$, pulmonary artery; $L A A$, left atrial appendage; $R A$, right atrium; $R V$, right ventricle; $L V$, left ventricle; $C S$, coronary sinus; $I V C$, inferior vena cava; $S V C$, superior vena cava.

was discharged from the intensive care unit on the 15th postoperative day and from the hospital 27 days after surgery. The patient remains asymptomatic and without signs of SVC syndrome in 2 years of follow-up. Endomyocardial biopsies were performed via the femoral approach. Echocardiogram showed normal heart function.

The technique used in this case was successful due to prior knowledge, and there was the possibility of planning. However, in cases in which the diagnosis is intraoperative, there are several techniques to help adult cardiac surgeons, who are less familiar with treating congenital diseases, to resolve this problem.

The use of the biatrial technique, preserving the recipient's coronary sinus, is technically a simple option that

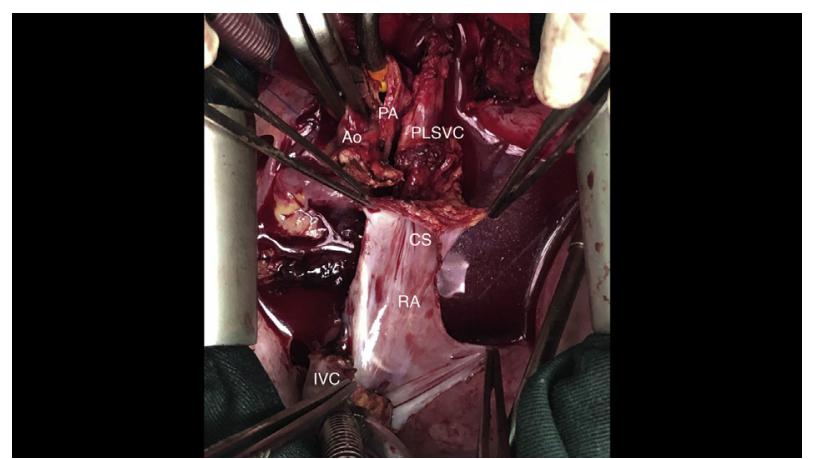

VIDEO 1. Clinical video of heart transplantation with persistent left vena cava showing intraoperative images and chest computed tomography 3dimensional reconstructions. Video available at: https://www.jtcvs.org/ article/S2666-2507(20)30285-6/fulltext. fits most patients with PLSVC. However, the documented superiority of bicaval technique in terms of postoperative arrhythmia, tricuspid valve regurgitation, and mortality suggests that efforts aimed at a bicaval anastomosis even in PLSVC situations may be warranted.

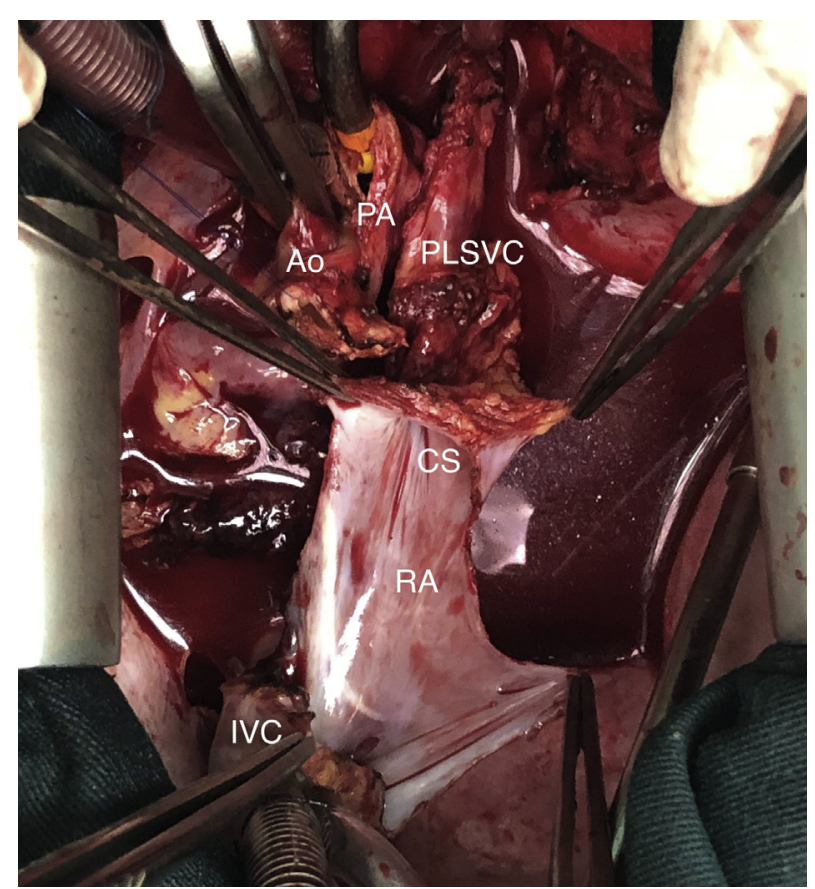

FIGURE 2. Heart transplantation with persistent left vena cava using the bicaval technique. Intraoperative photograph after explantation of the native heart showed drainage through the PLSVC to the CS. PA, Pulmonary artery; $A o$, aorta; $P L S V C$, persistent left superior vena cava; $C S$, coronary sinus; $R A$, right atrium; $I V C$, inferior vena cava. 
Other options may include reconstruction using autologous, ${ }^{3}$ or artificial grafts to extend the length of the PLSVC and perform its anastomosis on the right appendage or SVC. ${ }^{4}$ It is also possible to preserve PLSVC in situ by patching the inferior vena cava and the coronary sinus anastomosed in the donor inferior vena cava. However, these techniques require more time and surgical skills to obtain a satisfactory result. Besides, when artificial materials are used to reconstruct the venous system, the risk of infection in immunosuppressed patients increases. ${ }^{4}$

The patient in question had an extremely rare anatomical conformation in patients without situs inversus totalis. There was no right SVC, and the innominate vein made the venous drainage of the right upper body to the PLSVC (Figure 1, B).

This malformation was known before the surgical procedure, making it possible to incorporate a surgical strategy that enables a tension-free anastomosis between the donor's SVC and the recipient's PLSVC. The anterior position to the great vessels served as a facilitator to review the hemostasis (Figure 1,C). Computed tomography on the 30th postoperative day showed normal venous drainage through the PLSVC to the SVC (Figure 1,D).

In conclusion, it was possible to perform a simple surgical technique for a bicaval orthotopic heart transplantation without the use of prosthetic materials in a patient with persistent left superior vena cava.

\section{References}

1. Povoski SP, Khabiri H. Persistent left superior vena cava: review of the literature, clinical implications, and relevance of alterations in thoracic central venous anatomy as pertaining to the general principles of central venous access device placement and venography in cancer patients. World J Surg Oncol. 2011; 9:173.

2. Tanaka A, Lonchyna VA, Ota T. Persistent left superior vena cava and cardiac transplantation: anterior rerouting with anastomosis to the right atrium. J Heart Lung Transplant. 2015;34:1215-6.

3. Neragi-Miandoab S, Baran D, Godelman A, Goldstein DJ. Orthotopic heart transplantation in patients with persistent left superior vena cava: bicaval and biatrial techniques. Ann Thorac Surg. 2014;97:1085-7.

4. Lee JH, Park EA, Lee W, Cho HJ, Kim KB, Hwang HY. Heart transplantation in a patient with persistent left superior vena cava. Korean J Thorac Cardiovasc Surg. 2014; $47: 533-5$. 